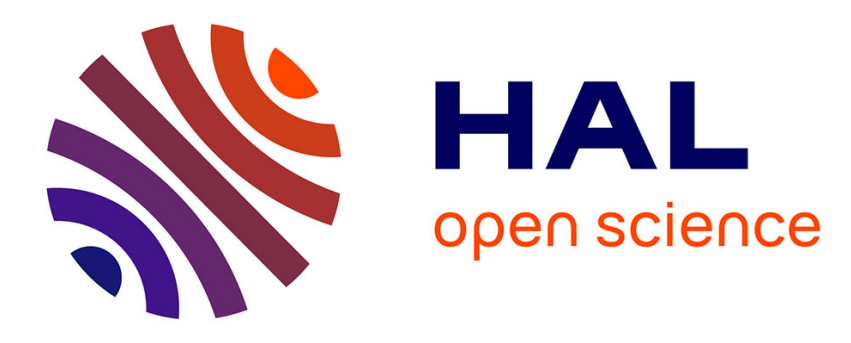

\title{
Autour de l'identité héraldique de la noblesse provençale au XIIIe siècle
}

Martin Aurell

\section{To cite this version:}

Martin Aurell. Autour de l'identité héraldique de la noblesse provençale au XIIIe siècle. Médiévales, 1990, 19, pp.17-27. 10.3406/medi.1990.1179 . halshs-00753745

\section{HAL Id: halshs-00753745 \\ https://shs.hal.science/halshs-00753745}

Submitted on 22 Apr 2016

HAL is a multi-disciplinary open access archive for the deposit and dissemination of scientific research documents, whether they are published or not. The documents may come from teaching and research institutions in France or abroad, or from public or private research centers.
L'archive ouverte pluridisciplinaire HAL, est destinée au dépôt et à la diffusion de documents scientifiques de niveau recherche, publiés ou non, émanant des établissements d'enseignement et de recherche français ou étrangers, des laboratoires publics ou privés. 


\section{Autour de l'identité héraldique de la noblesse provençale au XIIle} siècle

Monsieur Martin Aurell

\section{Citer ce document / Cite this document :}

Aurell Martin. Autour de l'identité héraldique de la noblesse provençale au XIlle siècle. In: Médiévales, n¹9, 1990. Liens de famille. Vivre et choisir sa parenté. pp. 17-27.

doi : 10.3406/medi.1990.1179

http://www.persee.fr/doc/medi_0751-2708_1990_num_9_19_1179

Document généré le 16/10/2015 
Médiévales 19, automne 1990, p. 17-27

Martin AURELL

\section{AUTOUR DE L'IDENTITÉ HÉRALDIQUE DE LA NObleSSE PROVENÇALE AU XIIIe SIÈCLE}

C'est autour du thème de l'identité que se déroulent actuellement les travaux du séminaire que Christiane Klapisch-Zuber anime à l'E.H.E.S.S. Les recherches et la réflexion des participants y portent sur les emblèmes, les signes et les marqueurs par lesquels un groupe social se définit en tant que tel ; ces symboles permettent de déceler la conscience que des individus manifestent d'être rattachés à un ensemble social et d'appréhender la nature des liens qui les unissent ${ }^{1}$. Grâce à la notion d'identité, les relations qu'entretiennent imaginaire collectif et sociabilité peuvent être envisagées sous un jour nouveau.

La parenté, forme la plus élémentaire de sociabilité ${ }^{2}$, est un champ privilégié de l'analyse de ces prises de position identitaires. Audelà de la filiation biologique, c'est la conscience généalogique qui pousse l'individu à se vouloir membre d'une famille, d'un lignage ou d'un cousinage ${ }^{3}$. Pour pénétrer cette réalité au sein de la noblesse médiévale, la patronymie ${ }^{4}$ comme l'héraldique $^{5}$ sont deux voies d'approche remarquables. Dans le bref espace qui nous est imparti, l'étude des armoiries devrait permettre d'effleurer cet aspect des mentalités aristocratiques et de nous interroger sur la conscience généalogique, les liens de sociabilité et les réseaux politiques de la noblesse provençale du XIII' siècle.

Quelque soixante sceaux, bulles ou matrices contenant des armoiries nobiliaires subsistent pour la Provence entre 1190 et $1300^{6}$. Pour

1. Ch. Klapisch-Zuber, "Ruptures de parenté et changement d'identité chez les magnats florentins du XIV siècle ", Annales E.S.C., 1988, pp. 1205-1240.

2. F. Thelamon et GrhIS - Dir. -, Aux sources de la puissance : Sociabilité et Parenté, Rouen, 1989.

3. G. DuBY, Hommes et structures du Moyen Age, Paris, 1973, pp. 287-298.

4. Cf. sur ce point, outre l'article cité de Ch. Klapisch, les pages très suggestives de D. BarthelemY, Les deux âges de la seigneurie banale, Paris, 1984, pp. 140 sq.

5. M. Pastoureau, "Stratégies héraldiques et changements d'armoiries chez les magnats florentins du xve siècle ", Annales E.S.C., 1988, pp. 1241-1256.

6. Le chercheur dispose de deux instruments de travail remarquables : L. BLAN- 
la plupart, ils représentent, à l'avers, un cavalier dont l'écu est armorié; leur revers reprend ces mêmes armes dans le champ : par un phénomène courant de dédoublement, l'image équestre aux armoiries miniaturisées et à peine lisibles trouve son pendant dans le contresceau qui contient une représentation exclusive et détaillée de ces mêmes armes (Figures 1 et 2).

En Provence, les exemples les plus précoces d'utilisation d'armoiries proviennent - l'on pourrait s'en douter - de la chancellerie comtale. En 1150, dans un sceau appendu à une charte provençale, les pals de la Catalogne ornent pour la première fois le champ de l'écusson d'un comte de Barcelone, en l'occurrence de Raimon Bérenger IV (1131-1162) ${ }^{7}$. A la suite des princes, l'aristocratie adopte cette pratique ; le premier cas d'héraldique nobiliaire provençale date d'environ 1170 : il s'agit du sceau apposé par Guilhem de Sabran, connétable de Raimon V de Toulouse (1149-1194), à un parchemin qui contient l'enquête sur l'albergue perçue par les comtes de Forcalquier à Apt ${ }^{8}$; son avers et son revers représentent le lion rampant des Sabran (Figure 1). Ces armoiries, originaires du comté de Forcalquier,

CARD, Iconographie des sceaux et bulles conservés dans la partie antérieure à 1790 des Archives Départementales des Bouches-du-Rhône, Marseille-Paris, 1860 et L. BARTHELEMY, "Sigillographie des diverses branches de la famille de Baux ", dans Inventaire chronologique et analytique des chartes de la maison de Baux, Marseille, 1882, pp. 549-564 et planches. Cf. également H. de Gerin-RICARD, E. ISNaRd, Actes concernant les vicomtes de Marseille et leurs descendants, Monaco-Paris, 1926, pp. LXV-LXVII ; A. Deloye, « Notice sur les sceaux de Géraud Adhémar, d'Aliette d'Ancezune et de Saint-Martin de Bollène ", Revue archéologique, T. II, 1845-1846, pp. 650-663 ; M. BABELON, communication sur une matrice trouvée à Roquemartine publiée dans le Bulletin archéologique du comite des travaux historiques et scientifiques, 1901, p. XXV ; J. RomaN, " Note sur la bulle de plomb de Bertrand et Raymond de Baux, co-princes d'Orange (1279-1330) » dans Bulletin de la société des antiquaires de France, 1913, pp. 174-176 ; L. DOUET D'ARCQ, " Rapport sur un sceau en plomb trouvé dans l'ancien manoir féodal dit la Tour de Sabran ", Revue des sociétés savantes (5e série), T. II, 1870 , pp. 77-78 ; A. de MarTonNe, "Histoire et sceau des seigneurs d'Hyères", Société de Sphragistique de Paris, T. III, 1853-1854, pp. 180-193. J.-Ph. Lagrue nous a aimablement communiqué une photo de la bulle des seigneurs de Fos, Archives Communales de Martigues, AA2 (1226). Nous ne connaissons qu'un seul exemple d'héraldique sculptée funéraire pour la noblesse provençale du xilre siècle : dessin édité par A. Ruffi au XVII' siècle et repris dans M. Aurell, La vielle et l'épée. Troubadours

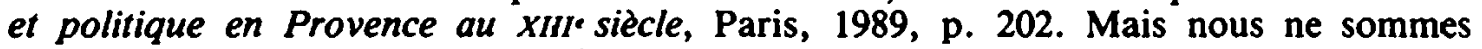
qu'au début de notre enquête et de nouvelles représentations armoriées devraient compléter ce corpus.

7. Blancard, Iconographie... pl. $2 \mathrm{n}^{\circ}$ 1. Cf. l'article dans lequel M. Pastoureau démontre les origines bourguignonnes de ces armoiries, réédité dans $L$ 'hermine et le sinople. Etudes d'héraldique médiévale, Paris, 1982, pp. 95-102.

8. Archives Départementales des Bouches-du-Rhône B 281. Ce document n'est pas daté, mais au cours de l'enquête qu'il contient l'un des témoins affirme avoir vu verser l'albergue quinze ans avant la mort d'un comte qui est, d'après le contexte, Guilhem ler (1090-1129). Ce témoin était donc en vie autour de 1115 : sa déposition ne saurait être postérieure aux années 1180 . En outre, Guilhem de Sabran porte pour la première fois le titre de connétable du comte de Toulouse en 1158 ; il disparaît de la documentation à partir de 1177 (E.-G. LEONARD, Catalogue des actes de Raimon $V$ de Toulouse (1149-1194), Nîmes, 1932, $\mathrm{n}^{\circ} 11$ et 84). Le Guilhem de Sabran qui apparaît dès 1184 et qui ne porte pas le titre de connétable est, sans doute, son fils. 
précèdent d'une vingtaine d'années le contresceau armorié d'Uc Sacristain-Porcelet, premier document original de l'héraldique nobiliaire du comté de Provence au sens strict (Figure 2$)^{9}$. Tout au long $\mathrm{du}$ XIII siècle, les plus en vue des nobles provençaux adoptent progressivement des armoiries ${ }^{10}$; autour de 1250 , quelque vingt familles détiennent leurs propres sceaux qui se retrouvent dans les chartes par lesquelles le comte Charles d'Anjou (1245-1285), représentant d'une nouvelle dynastie, entérine sa domination dans le comté.

En comparaison avec la France septentrionale ", la date de la naissance de l'héraldique dans nos principautés ne surprend guère : c'est au cours du second tiers du XII' siècle qu'apparaissent et se répandent les armoiries en Europe occidentale ${ }^{12}$. Cette chronologie coïncide avec la fin du lignage, son éclatement en plusieurs branches collatérales et la multiplication des maisons des cadets : le desserrement de l'étau lignager, loin d'effacer la mémoire généalogique de l'appartenance à une souche commune, semble la développer. Un autre trait de la sigillographie provençale paraît, en revanche, plus original : son caractère élitiste. Outre le comte et les puissantes communes indépendantes, seule la très haute noblesse utilise des sceaux armoriés ${ }^{13}$; la plupart des quelque vingt familles qui arborent des armes sont issues du groupe des nobles de vieille souche; l'héraldique est encore l'affaire des domini et des nobiles plutôt que des milites ${ }^{14}$.

Le nombre de figures que contiennent ces armoiries est très limité. L'étoile, qui apparaît dans un quart des sceaux étudiés, est le signe le plus usité (Figure 3). Ainsi, l'étoile aux seize raies est l'emblème par excellence de la tentaculaire maison de Baux qui en fait l'élément clef d'armoiries parlantes: "Au hasard Bal-thazar!" 15, phrase héraldique de cette famille, renvoie à l'épisode des rois mages à la poursuite de l'astre ; elle se rapporte à l'Orient où, depuis la fin du

9. A. RuffI, historien du XVIre siècle, décrit quelques sceaux aujourd'hui disparus de Raimon Jaufre et de Barral, vicomtes de Marseille à la fin du XIle siècle, cf. GERIN-RICARD, ISNARD, Actes... pp. LXV-LXVII.

10. Voici la distribution chronologique des quarante sceaux nobiliaires édités par L. BLANCARD : 2 pour la période $1190-1199 ; 6$ pour $1200-1209 ; 3$ pour $1210-1219 ; 4$ pour $1220-1229$; 5 pour $1230-1239 ; 6$ pour $1240-1249 ; 9$ pour $1250-1259$ et 4 pour $1260-1300$.

11. M. Pastoureau, Figures et couleurs. Etude sur la symbolique et la sensibilité médiévales, Paris, 1986, p. 89.

12. Sur la fractionnement du lignage et l'organisation de la famille autour du rameau, cf. le récent article d'O. GUYOTJEANNIN, "Lignage et mémoire généalogique en Emilie au XIIr siècle : l'exemple de Salimbene de Adam ", Melanges $K$. F. Werner, Paris, 1989, pp. 225-241.

13. Cela contraste fortement avec la Normandie, la Flandre et l'Ile-de-France où les sceaux armoriés de paysans sont nombreux. En Normandie, Bretagne et Poitou, les simples écuyers et les damoiseaux utilisent de même des emblèmes héraldiques, PAS. TOUREAU, Figures... pp. 96-97.

14. Parmi les familles récentes, celles notamment de ministériels de la cour d'origine catalane, seuls les Villeneuve utilisent des sceaux armoriés au xiIt siècle, BLANCARD, Iconographie... pl. $30 \mathrm{n}^{\circ} 7$.

15. Bals et Baux sont phonétiquement interchangeables au xIIt* siècle. 
$\mathrm{XI}^{\mathrm{e}}$ siècle, des membres de cette maison, installés dans le comté de Tripoli, participaient à la croisade, enrichissant le légendaire familial d'exploits à l'authenticité incertaine, mais qui devenaient un élément important pour - sans mauvais jeu de mots - redorer le blason des membres de la famille restés en Provence ${ }^{16}$. Les Baux ne réservaient pas l'étoile à un usage strictement sigillographique ; une enquête de 1269 sur la façon dont ils exerçaient la justice dans leur tribunal de Trinquetaille nous apprend qu'elle faisait partie de leur attirail répressif : l'étoile était marquée au fer rouge sur le front de certains voleurs ${ }^{17}$; l'emblème de la famille contribuait d'une bien terrifiante façon à faire respecter la justice seigneuriale.

Les Baux ne détiennent toutefois pas l'exclusivité de l'étoile aux seize raies. Les Blacas-Beaudinar, seigneurs de vastes domaines en Provence centrale, leur ont emprunté cette figure qu'ils utilisent habituellement dans leur sceau (Figure 4). Or, aucun lien familial direct ne semble unir ces deux familles ${ }^{18}$. La politique des deux maisons n'a d'ailleurs guère joué dans le même sens aux XII ${ }^{e}$ et XIII ${ }^{e}$ siècles ; l'on ne saurait donc expliquer ces emprunts par une quelconque insertion des Blacas dans les clientèles des Baux : les deux familles appartiennent au même groupe de la haute aristocratie et leurs domaines sont trop éloignés pour songer à une dépendance des uns envers les autres. $\mathrm{Ni}$ la parenté ni la vassalité ne rendent compte de l'adoption des armoiries des Baux par les Blacas ${ }^{19}$. Il en va de même pour les familles d'Amic et de Simiane qui font leurs, sans aucun motif apparent, les armoiries parlantes des Castellane : un château fermé, crénelé, maçonné à trois tours dont celle du milieu est la plus haute (Figures 5 et 6). Pourtant, l'éloignement des domaines de ces maisons ne pouvait être plus important.

Un semblable mimétisme apparaît chez les vicomtes de Marseille qui se parent, au moins dès 1201 , de la croix pommetée et cléchée de Toulouse ${ }^{20}$. Cependant, c'est avec une constance sans faille que

16. J.-P. Poly, La Provence et la société féodale (879-1166), Paris, 1976, p. 358.

17. "vidit quemdam hominem extraneum cujus nomen nescit fustigari per villam Trencatallarum portando gallum in collum quia dicebatur substraxisse gallinas de primodo ante hospitale pauperum quod est juxta reclusa. Fuit dictus homo decoctus in fronte cum quodam ferro rotundo quo affilantur stella ", Archives Départementales des Bouches-du-Rhône B $1069 \mathrm{f}^{\circ} 95$.

18. C'est du moins ce qui ressort de la documentation - fragmentaire, il est vrai - concernant ces deux familles. Comme chez les Baux, bon nombre des membres de cette maison s'appellent Raimon, mais c'est là un prénom très répandu dans l'aristocratie provençale et son adoption ne permet pas de conclure $a$ un quelconque lien de parenté. Il n'en reste pas moins qu'encore de nos jours les descendants des Blacas attribuent le hâle de leur peau à des origines africaines; cet élément de la mémoire familiale ne manque pas d'intérêt : peut-être se rapporte-t-il au roi Balthazar des Baux par les jeux d'un légendaire de la manipulation généalogique dont il est difficile de discerner les étapes ?

19. Semblable sur ce point, aussi inexplicable apparaît le comportement de Boniface de Castellane (1195-1252), donnant à son second fils le nom d'Uc de Baux, Aurell, La vielle... p. 179.

20. Cf. Gerin-Ricard, ISnard, Actes concernant... pp. LXV-LXVII, en dépit de 
les Marseille choisissent, tout au long du XIre siècle, le camp des comtes de Barcelone dans la guerre qui oppose ces derniers aux représentants de la maison de Toulouse. Au cours de la première moitié du XIII siècle, ils tournent leurs armes contre la commune de Marseille dont le recteur n'est autre que Raimon VII (1222-1249). Comme dans l'exemple précédent, l'attitude politique des vicomtes de Marseille semble contraire aux figures héraldiques qu'ils arborent sur un champ de bataille où ils croisent souvent les armes avec le détenteur attitré de la croix pommetée.

Aussi paradoxale soit-elle, cette attitude d'admiration envers l'ennemi se retrouve chez les nobles provençaux qui composent des sirventes, chansons politiques. Bertran de Lamanon (1210-1270), fidèle ministériel au service de Raimon Bérenger $\mathrm{V}$ aux jours les plus mouvementés de la guerre entre son maître et Raimon VII, chante dans ses poèmes le comte de Toulouse : Raimon VII, dernier représentant d'un monde sans Etat qu'il regrette avec nostalgie, incarne à ses yeux toutes les valeurs chevaleresques; dans ses sirventes l'ennemi invétéré de son maître apparaît comme plazen (" agréable ») et de bel estatge (" de haut rang ") : il restaure paratge (" noblesse »), gaug (" joie "), solatz (" société ») et pretz (" mérite »), toutes vertus qui se perdaient en Provence ${ }^{21}$. Les contradictions de ce chevalier, dont nous connaissons les prises de position politiques grâce à ses chansons, nous éclairent sur les emprunts héraldiques des vicomtes de Marseille.

Le lion, rampant ou passant, est la figure animalière la plus usitée dans l'héraldique nobilaire provençale du XIII' siècle ${ }^{22}$. Comme nous l'avons vu, c'est l'emblème des Sabran dont les membres appartiennent au parti toulousain (Figure 1). Le même choix politique se retrouve chez Gui de Cavaillon, viguier de Raimon VI pour le Venaissin et l'un des plus fidèles conseillers de Raimon VII, qui est également un adepte du lion : nous ne conservons aucun de ses sceaux, mais nous savons que cet animal ornait le champ de ses armes grâce aux vers que ce troubadour échangea en 1216 avec Guilhem de Baux, son ennemi, qui le tournait en dérision dans ces termes : "Domestiquez votre lion qui est un tout petit trop en colère ; cela ne nous profiterait en rien s'il nous mangeait tous " ; dans le sirventes que Bertran Folco envoya à Gui en 1221 pour l'encourager à tenir bon dans la ville de Castelnaudary assiégée par Amaury de Montfort, il lui était

l'insistance, toute servile, pour vouloir octroyer à tout prix des armes propres à cette maison, minimisant la portée de l'adoption de l'emblème héraldique des comtes de Toulouse. Les comtes de Forcalquier, alliés traditionnels des Toulousains, portent également la croix pommetée dans leurs armes.

21. AURELl, La vielle... p. 135.

22. Il apparaît dans six des quarante sceaux aristocratiques armoiriés dessinés par L. BLANCARD, Iconographie... et M. PASTOUREAU calcule que dans cette principauté il apparaît dans plus de la moitié des armoiries animalières, PASTOUREAU, L'hermine... p. 22. Sur ce point la Provence ne présente aucune originalité : " le lion est de tous les meubles celui qui a été le plus employé par l'héraldique médiévale ", Ibidem p. 108. 
au contraire recommandé de "pousser son lion vers les Français » ${ }^{23}$. Cette figure fut également portée par les Fos $^{24}$ qui l'abandonnèrent pour le plus prosaïque cochon de la maison de Porcelet sans doute à la suite du traité de mars $1188 / 89$ qui mettait fin à la guerre privée entre ces deux maisons et établissait leur coseigneurie sur le castrum de $\operatorname{Fos}^{25}$ : tout au long du XIII' siècle, de nombreux mariages unissent ces deux familles et c'est par les femmes que la figure héraldique des Porcelet a été transmise aux Fos ${ }^{26}$. Contrairement au patronyme, les règles de la patrilinéarité ne sont pas contraignantes en héraldique, où les armes de la famille maternelle évincent souvent celles des ancêtres par voie agnatique.

Aussi surprenante soit-elle, l'utilisation du porcelet - dont la morphologie médiévale rappelle plus le sauvage sanglier que nos gras cochons - est fort répandue dans l'héraldique médiévale ${ }^{27}$. Au sein de la famille Porcelet, cette figure donna lieu à de nombreuses élucubrations et élaborations légendaires. Les généalogistes du XVII siècle font remonter cette maison à Diego Porcellos, prétendu comte de Castille, dont la mère accoucha de neuf garçons à la suite des imprécations d'une femme à qui la comtesse refusa l'aumône et qui, en guise de vengeance, souhaita qu'elle accouchât d'autant d'enfants qu'il y a de porcelets dans la portée d'une truie ${ }^{28}$. Cette anecdote, transmise certes par une documentation tardive, reprend assurément des traditions orales qui avaient cours à des périodes plus anciennes, médiévales sans doute : elle se rapporte au mythe de la fécondité auquel les porcins sont universellement rattachés. La légende de cet accouchement prodigieux, fondateur de la famille Porcelet, devait accrô̂tre le prestige de ses membres auprès de leurs contemporains.

Un dernier meuble animalier - l'aigle - est assez répandu dans l'héraldique provençale. On pourrait le croire étroitement lié au parti impérial, dont les adeptes sont nombreux en Provence au cours de la première moitié du XIII siècle, et à la prise de positions politiques imprégnées de gibelinisme ${ }^{29}$. Le gerfaut, puis l'aigle, des armes de la

23. Aurell, $L a$ vielle... pp. 42, 68, 253-256 et 298 n. 16.

24. BLANCARD, Iconographie... pl. $30 \mathrm{n}^{\circ} 8$ et MARTONNE, "Histoire et sceau..." "

25. AURell, Une famille de la noblesse provençale au Moyen Age: les Porcelet, Avignon, 1986, p. 65 .

26. Cf. la description du sceau au porcelet de Giraud et Peire Raimon de Fos apposée à une charte du 5 août 1215, aujourd'hui perdue, dans Bibliothèque d'Arles, manuscrit $882 \mathrm{f}^{\circ} 3$. La bulle de 1226 des Archives Communales de Martigues, AA2, représente dans le champ un cochon passant à droite surmonté de trois étoiles à six raies; la légende est + SIGIL. DOMINORUM CASTRI DE FOS.

27. M. Pastoureau, "Le sanglier dans les sceaux du Moyen Age ", Le bestiaire des monnaies, des sceaux et des médailles. Catalogue de l'exposition de l'Hôtel de la monnaie, Paris, juin-septembre 1974, reédité dans L'hermine...

28. Même si dans une perspective étroitement positiviste elles paraissent incongrues et puériles, les anecdotes rapportées par les généalogistes de l'époque moderne au sujet des familles nobiliaires renvoient à un imaginaire familial plus ancien qui est riche en éléments folkloriques ; elles mériteraient un récensement et une analyse systématiques.

29. Sur le gibelinisme en Provence, cf. les recherches en cours de J. ChiffoleaU 
commune d'Avignon, en seraient le meilleur exemple : c'est dans cette ville, acquise au comte de Toulouse, que les vicaires de Frédéric II trouvèrent les plus solides de leurs appuis. Cela avait poussé quelques érudits à insister sur le rôle politique de l'adoption de l'aigle par les consuls avignonnais, férus gibelins dévoués à l'Empereur. Leur point de vue ne résista pas à la critique de L.-H. Labande qui minimisa, voire annulla, de façon fort convaincante, le lien de cause à effet entre triomphe du parti impérial en Avignon et impression de l'aigle sur la bulle des consuls ${ }^{30}$.

Cette figure animalière apparait également dans un seul des sceaux de Barral de Baux (1217-1268), podestat d'Avignon et gibelin notoire, la personnalité politique provençale la plus en vue de son temps (Figure 7). Une empreinte sur cire jaune où quatre aigles cantonnent la croix de Toulouse valide une de ses chartes de novembre 1251. Or, cette date est d'une année postérieure à la trahison de Barral envers le parti impérial; en mars 1250, il avait, en effet, abandonné ses alliés d'antan pour conclure un pacte avec Blanche de Castille et passer au camp capétien. Une fois de plus, force est de constater que l'adoption de l'aigle héraldique ne correspond pas nécessairement à des prises de position gibelines. En fait, les armoiries de Barral, descendant des vicomtes de Marseille et des Baux, sont plutôt à la croix cléchée et pommetée et à l'étoile de ses ancêtres; l'utilisation de ces figures dans le champ de ses contresceaux ne semble, à une époque de flottement héraldique ${ }^{31}$, nullement fixée ; c'est de façon arbitraire qu'elles sont gravées sur les matrices (Figures 8 et 9). Ce large choix d'armoiries ne saurait, en tout état de cause, s'expliquer par les revirements politiques de celui qui fut le fidèle de Raimon VII, de Frédéric II et des communes indépendantes du Bas-Rhône, avant de devenir le serviteur dévoué de Charles d'Anjou dans son expansion provençale et napolitaine.

Ce rapide survol de l'héraldique nobiliaire du XIII siècle provençal nous permet d'avancer une hypothèse de travail que confirmeront ou infirmeront d'ultérieures recherches. L'on sait depuis longtemps que les signes héraldiques, qui se vulgarisent au cours des décennies du sommet à la base, se diffusent à l'instar d'un modèle culturel ${ }^{32}$. Les

et ses deux articles : "Les mendiants, le prince et l'hérésie à Marseille vers 1260 », Provence Historique, 1986, pp. 3-19 et « Vie et mort de l'hérésie en Provence et dans la vallée du Rhône du début du XIII" au début du XIve siècle ", Cahiers de Fanjeaux, T. XX, pp. 73-99.

30. "En somme, en transformant au revers de leur bulle l'ancien gerfaut en aigle, on s'inspira tout simplement d'usages déjà implantés dans le pays ", Avignon au XIII' siècle, Paris, 1908 (réédition anastatique, Marseille, 1975) p. 285.

31. " Pour le Moyen Age, en effet, toutes les études mettent en valeur le caractère extrêmement flottant des règles du blason, la grande part d'incertitude réservée aux artistes et l'absence d'un véritable système de brisure " PASTOUREAU, L'hermine... p. 6.

32. G. Duby, Hommes et structures... p. 303 et M. Pastoureau, Les armoiries, Turnhout, 1976, p. 27. 
figures des armoiries étudiées se répandent d'après ce même schéma au sein de notre groupe social : c'est aux familles les plus prestigieuses - comtes de Toulouse ou Baux - que les maisons nobiliaires empruntent la croix ou l'étoile, signes les plus fréquemment portés. Ce phénomène de mimétisme ne répond pas toujours à des raisons politiques ni familiales : les paradoxes de la fascination pousseront tel noble à arborer sur son écu les armes de son ennemi de toujours ; il le fera d'autant plus volontiers qu'elles comportent des légendes et anecdotes aptes à rehausser le prestige de sa maison. En imitant un grand aristocrate qui, comme Raimon VII aux yeux de beaucoup, incarne mieux que quiconque les valeurs chevaleresques, le noble tentera de surpasser ses frustrations au cours d'un siècle où les mutations de son groupe social, impuissant face à la montée de l'Etat, le plongent dans les nostalgies d'une monde perdu à jamais. Pour ce qui est de l'héraldique, identité et identification vont de pair.

Comme le nom de famille, les armoiries jouent un autre rôle psychologique. Elles permettent de perdurer au lignage menacé d'extinction biologique. Cette survie identitaire saute particulièrement aux yeux de l'historien qui rencontre, au détour d'un testament, les instructions laissées par un aristocrate moribond au sujet de la transmission de ses armes. L'exemple le plus précoce que nous connaissions en Provence date du 23 janvier 1310/1311 ; c'est dans ces termes que Guilhem Porcelet, seigneur de Cabriès, institue héritier universel son neveu Rainaudet, fils de Giraud d'Agoult : " Je veux et commande que mon héritier Rainaudet porte mon patronyme, à savoir Porcelet, et les armes ou le signe des Porcelet : qu'il grave un cochon de sable sur son écu, son cellier et son sceau. A sa mort, je veux que ses héritiers fassent de même successivement jusqu'à l'infini " ${ }^{33}$. Au creuset de l'imaginaire, l'héraldique est l'enclume où se forge la conscience généalogique.

33. «Volens et mandans quod dictus Raynaudetus, heres meus, habeat, quantum in ipso erit, cognomen meum, videlicet Porcelleti, et arma seu signum Porcelletorum, videlicet porcum nigrum faciat in scuto, cella et sigillo suo. Post vero mortem suam volo quod idem faciant ejus heredes successive in infinitum. "Archives Départementales des Bouches-du-Rhône, 5 G $20 \mathrm{n}^{\circ}$ 142, vidimus du $29 \times 1325$. 
REPRÉSENTATIONS DE SCEAUX

Figure 1 - Sceau de Guilhem de Sabran, entre 1158 et 1177 : + SIGILLVM GVILELMI DE SABRANO DONI COMES.TABULUS

Figure 2 - Sceau d'Uc Sacristain-Porcelet, mars 1190 : + SIGILLVM VGONIS SACRISTAN

Figure 3 - Sceau d'Uc de Baux, vicomte de Marseille, 13 août 1214 : + 
Figure 4 - Sceau de Blacas d'Aups, août 1233 : + SIGILLVM. BLACHASSII. DOMINI DE ALMIS.

Figure 5 - Sceau de Boniface de Castellane, 1205 : + SIGILLVM : BONI-

FACII : DE : CASTELLANA :

Figure 6 - Sceau de Giraud Amic, mai 1221 : + SIGILL'. G. AMICI 
Figure 7 - Sceau de Barral de Baux, 19 novembre 1251 : + SIGILLVM : BARRALI : DOMINI : BAVCII :

Figure 8 - Bulle de Barral de Baux, 7 juin $1254:+: S:$ DNI : BARALIS DE BAVSCIO + SIGILL' DNI BARRALIS DE BAUX : CIO :

Figure 9 - Bulle de Barral de Baux, non datée : + BVLLA : BARRALI D BAVCIO D MONTILLIS + S' BARALI. D BAVCIO. DNI D MONTILIS

(Dessins extraits de BlANCARD, Iconographie... et de LeONARD, Inventaire...) 